\title{
Modelling of Phase Structure and Stress State of the Rod from the Shape Memory Material at Thermomechanical Loading
}

\author{
Volodymyr Astashkin ${ }^{1}$, Oleksii Onyshko ${ }^{1}$ \\ Bohdan Bozhenko ${ }^{2,3}$ and Stepan Budz ${ }^{1}$ \\ ${ }^{1}$ Pidstryhach Institute for Applied Problems of Mechanics and Mathematics NASU \\ Naukova Str. 3-b, 79060 Lviv, Ukraine \\ e-mail:budz@iapmm.lviv.ua \\ ${ }^{2}$ Mathematical Modelling Center of IAPMM NASU \\ Dudayev Str. 15, 79005 Lviv, Ukraine \\ ${ }^{3}$ Opole University of Technology, ul. Luboszycka 3, 45-036 Opole, Poland \\ e-mail:b.bozhenko@po.opole.pl
}

(Received: 14 May 2008; published online: 19 November 2008)

\begin{abstract}
On the basis of the mathematical model proposed before, the phase and stress state of the shape memory rod during torsion and following heating has been determined. Non-uniform distribution of residual stresses induced by a change of the phase structure under loading decreases after heating due to material returning to the initial phase state.
\end{abstract}

Key words: mathematical model, shape memory material, phase and stress state

\section{INTRODUCTION}

Stress and strain tensors are common characteristics used in thermomechanical models for a quantitative description of deformation and heat transfer in solids. Such description is convenient if changes of parameters of nonmechanical processes in a system (concentration, phase structure, temperature) are caused by volume change only. At the same time, it is known that there are such processes in solids that essentially depend not only on the change of volume (hydrostatic pressure), but also on the change of the volume of the element shape related to the second invariant of stress and strain tensors. This holds true mainly for the phase transformations caused by shear stresses.

The behaviour of solids with the above-mentioned changes of internal structure is expedient to describe using the invariants of stress and strain tensors which can be taken as independent state parameters [1]. The invariant approach allows taking separately into account the influence of both spherical (volume change) and deviator (shape change) parts of stress and strain tensors on the phase structure of the system.
On the basis of the above-stated reasons a macroscopic thermodynamic model for the quantitative description of deformation of isotropic solids has been formulated. In the model the phase transformations caused by the influence of tension-compression and shear are described [2-4]. In doing so, the well-known methods of solid mechanics and thermodynamics of irreversible processes were used.

In the present work the behavior of the circular rod made of shape memory alloy is investigated with the use of a corresponding mathematical model. The martensite phase is first formed in the rod due to shear stresses. Subsequent heating causes martensite to be transformed into austenite.

\section{MODEL PRINCIPLES}

The used thermodynamic approach is based on the hypothesis of thermodynamic equilibrium. Like the state parameters describing thermal processes, absolute temperature $T$ and specific entropy $S$ are chosen. Martensitic transformation is characterized by martensite content $\Xi$ and specific affinity of transformation $A$ [5]. The me- 
chanical state related to volume change is described by invariants of stress and strain tensors $\sigma$ and $e$, respectively; that related to shape change is described by invariants $\sigma_{i}$ and $e_{i}[1]$, where

$\sigma=\frac{\Pi_{1}}{3}$ is the average hydrostatic pressure;

$e=\frac{I_{1}}{3}$ is the relative change of average linear sizes;

$\sigma_{i}=\sqrt{3 \Pi_{2}-\Pi_{1}^{2}} / \sqrt{2} ; e_{i}=\sqrt{3 I_{2}-I_{1}^{2}} / \sqrt{2}$ is the stress and strain intensities, respectively;

$\Pi_{1}=\sigma_{11}+\sigma_{22}+\sigma_{33}$ is the 1-st invariant of stress tensor;

$I_{1}=\varepsilon_{11}+\varepsilon_{22}+\varepsilon_{33}$ is the 1-st invariant of strain tensor;

$\Pi_{2}=\sigma_{11}^{2}+\sigma_{22}^{2}+\sigma_{33}^{2}+2\left(\sigma_{12}^{2}+\sigma_{13}^{2}+\sigma_{23}^{2}\right)$ is the 2-nd invariant of stress tensor;

$I_{2}=\varepsilon_{11}^{2}+\varepsilon_{22}^{2}+\varepsilon_{33}^{2}+2\left(\varepsilon_{12}^{2}+\varepsilon_{13}^{2}+\varepsilon_{23}^{2}\right)$ is the 2-nd invariant of strain tensor.

Initially the material of a rod is characterized by a certain initial value of the relative martensite content $\Xi=\Xi_{0}$ corresponding to the initial value of specific affinity of transformation $A_{0}$. Value $\Xi$ changes due to thermal and mechanical influences. Free energy $F=F\left(e, e_{i}, T, \Xi\right)$ is chosen as a function of the thermodynamic state. The generalized Gibbs equation with the use of invariants of stress and strain tensors is modified as

$$
d F=-S d T+\frac{3}{\rho} \sigma d e+\frac{1}{\rho} \sigma_{i} d e_{i}+A d \Xi .
$$

Here $\rho$ is the density of material.

Expanding free energy $F$ in power series in case of a small deviation from the initial equilibrium non-strained state $T=T_{0}, \quad S=S_{0}, \quad e=0, \quad \sigma=0, \quad e_{i}=0, \quad \sigma_{i}=0, \quad \Xi=\Xi_{0}$, $A=A_{0}$ and observing second order terms and also considering the Gibbs Eq. (1), the following system of linear equations of the state is found:

$$
\begin{gathered}
S=S_{0}+\frac{c_{t}}{T_{0}} t+\frac{1}{\rho} K \alpha e-K_{t \xi} \xi, \\
3 \sigma=K(e-\alpha t-\beta \xi), \\
\sigma_{i}=G\left(e_{i}-\beta^{\prime} \xi\right), \\
A=A_{0}+K_{\xi} \xi+K_{t \xi} t-\frac{1}{\rho} K \beta e-\frac{1}{\rho} G \beta^{\prime} e_{i},
\end{gathered}
$$

where $t=T-T_{0}, \quad \xi=\Xi-\Xi_{0}$.

The coefficients in (2) describe certain physical properties of model material:

$c_{t}$ is the specific heat;

$K$ is the comprehensive pressure modulus;

$G$ is the shear modulus; $\alpha$ is the temperature coefficient of volumetric expansion;

$K_{t \xi}$ is the coefficient of dependence of martensitic transformation specific affinity on temperature;

$\beta$ is the coefficient of volume change due to martensitic transformation;

$\beta^{\prime}$ is the coefficient of shape change due to martensitic transformation;

$K_{\xi}$ is the coefficient of dependence of martensitic transformation specific affinity on martensite content.

In the following, the material characteristics pertinent to the transformation of austenite into martensite will be denoted with index " $M$ ", while those describing the reverse transformation will be denoted with index " $A$ ".

\section{CIRCULAR ROD}

\section{III.1. Torsion}

Consider a long circular rod of radius $R$ made of shape memory alloy and loaded with moment of torsion $M$. It is accepted that in initial conditions the material of the rod stays in austenite (high-temperature) phase (martensite content $\Xi=0)$. Rod temperature $T=T_{1}$ is constant and falls in the interval where even insignificant stresses can cause the creation of martensite [5]. Let us determine the phase state of the rod formed under such loading.

It is known that such loading in the cylindrical system of coordinates with axis $z$ directed along the rod axis leads to only one component of stress tensor $\sigma_{\theta z}$ being nonzero:

$$
\sigma_{\theta z}=\frac{2 M r}{\pi R^{4}}
$$

The conditions for martensite transformation to take place are determined from the condition for a minimum of free energy $F$ under martensite content $\Xi$ at the fixed values of deformation and temperature [5]. For the problem discussed it takes the form

$$
K_{\xi}^{M} \Xi-\frac{E \beta_{M}^{\prime}}{3(1+v) \rho} e_{i}=0,
$$

where $E$ is the Young modulus, and $v$ is the Poisson ratio.

Using condition (4) and the 3-rd Eq. (2), and taking into account Eq. (3) the martensite distribution along rod radius $r$ is found:

$$
\Xi^{*}=\frac{4 \sqrt{3}(1+v) \beta_{M}^{\prime} M r}{\pi R^{4}\left[2(1+v) \rho K_{\xi}^{M}-E\left(\beta_{M}^{\prime}\right)^{2}\right]}
$$


If we remove the applied loading, the reached phase structure in the rod (5) is observed $(M=0)$. This holds true for materials with a wide enough hysteresis loop, that is, for the materials that do not undergo reverse transformation without additional external loadings [5-7]. The residual stresses caused by phase transformation are generated in the rod because the transformation of material from austenite to martensite is accompanied by the volumetric effect $[6,7]$. In the present case only one nonzero stress component survives

$$
\sigma_{r}^{*}=\frac{2 \sqrt{3}(1+v) \beta_{M}^{\prime}\left(\beta_{M}^{\prime}-2 \beta_{M}\right) M r}{\pi R^{4}(2-7 v)\left[2(1+v) \rho K_{\xi}^{M}-E\left(\beta_{M}^{\prime}\right)^{2}\right]}
$$

\subsection{Heating}

Now consider the change of phase structure (5) and stress state (6) of the rod after it is uniformly heated from temperature $T_{1}$ up to $T_{2}$, and free from mechanical loading. The condition for the reverse martensite transformation in this case takes the form

$$
K_{\xi}^{A} \Xi+K_{t \xi}^{A} t+\frac{E \beta_{A}}{3(1-2 v) \rho} e+\frac{E \beta_{A}^{\prime}}{3(1+v) \rho} e_{i}=0 /,
$$

where $t=T_{2}-T_{1}, \quad \xi=\Xi-\Xi^{*}$.

Using the state equations (2) and condition (7), the distributions of martensite

$$
\Xi=\Xi^{*}-\frac{D_{0} K_{\xi}^{A}-E D_{1} D_{2} \beta_{M}^{\prime}}{D_{0} K_{t \xi}^{A}-\alpha E D_{1} D_{2}} \cdot t \cdot \frac{r}{R}
$$

and residual stresses

$$
\sigma_{r}=\sigma_{r}^{*}-\frac{E}{4-7 v}\left[\alpha t+\left(\beta_{A}-\frac{1}{2} \beta_{A}^{\prime}\right) \xi\right]
$$

are obtained. Here:

$$
\begin{gathered}
D_{0}=9 \rho(1-2 v)(1+v)(7 v-2), \\
D_{1}=(1+v) \beta_{A}+(1-2 v) \beta_{A}^{\prime}, \\
D_{2}=2(1+v) \beta_{A}-3(1-2 v) \beta_{A}^{\prime} .
\end{gathered}
$$

\section{III.3. Calculations}

On the basis of the developed model the distributions of martensite and residual stresses in a rod are calculated for several known shape memory alloys. Fig. 1 illustrates the distribution of martensite in the rod with radius $R=10 \mathrm{~cm}$ caused by the moment of torsion $M=50 \mathrm{kN} \cdot \mathrm{m}$. The distribution of residual stresses due to martensitic transformation is shown in Fig. 2. The distributions of martensite and stresses after heating $(t=10 \mathrm{~K})$ are presented in Fig. 3 and Fig. 4, respectively.

Line 1 corresponds to alloy NiTi:

$\rho=6850\left[\mathrm{~kg} / \mathrm{m}^{3}\right], v=0.36, E=116[\mathrm{GPa}]$,

$\beta_{M}=0.5 \cdot 10^{-3}, \beta_{M}^{\prime}=2 \cdot 10^{-3}, K_{\xi}^{M}=50\left[\mathrm{~m} / \mathrm{s}^{2}\right]$,

$\alpha=5 \cdot 10^{-4}[1 / \mathrm{K}], \beta_{A}=-0.5 \cdot 10^{-3}, \beta_{A}^{\prime}=-3 \cdot 10^{-3}$,

$K_{\xi}^{A}=-10\left[\mathrm{~m}^{2} / \mathrm{s}^{2}\right], K_{t \xi}^{A}=-10^{-3}\left[\mathrm{~m}^{2} /\left(\mathrm{s}^{2} \cdot \mathrm{K}\right)\right] ;$

line 2 corresponds to alloy $\mathrm{NiAl}$ :

$\rho=7500\left[\mathrm{~kg} / \mathrm{m}^{3}\right], v=0.31, E=173[\mathrm{GPa}]$,

$\beta_{M}=0.8 \cdot 10^{-3}, \beta_{M}^{\prime}=2 \cdot 10^{-3}, K_{\xi}^{M}=50\left[\mathrm{~m}^{2} / \mathrm{s}^{2}\right]$,

$\alpha=10^{-4}[1 / \mathrm{K}], \beta_{A}=-0.8 \cdot 10^{-3}, \beta_{A}^{\prime}=-2 \cdot 10^{-3}$,

$K_{\xi}^{A}=-50\left[\mathrm{~m} / \mathrm{s}^{2}\right], K_{t \xi}^{A}=-10^{-3}\left[\mathrm{~m}^{2} /\left(\mathrm{s}^{2} \cdot \mathrm{K}\right)\right]$;

line 3 corresponds to alloy $\mathrm{CuAlNi}$ :

$\rho=8050\left[\mathrm{~kg} / \mathrm{m}^{3}\right], v=0.36, E=110[\mathrm{GPa}]$,

$\beta_{M}=0.3 \cdot 10^{-3}, \beta_{M}^{\prime}=10^{-3}, K_{\xi}^{M}=30\left[\mathrm{~m}^{2} / \mathrm{s}^{2}\right]$,

$\alpha=3 \cdot 10^{-4}[1 / \mathrm{K}], \quad \beta_{A}=-0.3 \beta_{A}^{\prime}=-10^{-3} \cdot 10^{-3}$,

$K_{\xi}^{A}=-30\left[\mathrm{~m}^{2} / \mathrm{s}^{2}\right], K_{t \xi}^{A}=-10^{-3}\left[\mathrm{~m}^{2} /\left(\mathrm{s}^{2} \cdot \mathrm{K}\right)\right]$;

line 4 corresponds to alloy $\mathrm{CuZn}$ :

$\rho=7920\left[\mathrm{~kg} / \mathrm{m}^{3}\right], \quad v=0.31, E=120 \mathrm{GPa}$,

$\beta_{M}=0.4 \cdot 10^{-3}, \beta_{M}^{\prime}=1.4 \cdot 10^{-3}, K_{\xi}^{M}=30\left[\mathrm{~m}^{2} / \mathrm{s}^{2}\right]$,

$\alpha=2 \cdot 10^{-4}[1 / \mathrm{K}], \quad \beta_{A}=-0.4 \cdot 10^{-3}, \quad \beta_{A}^{\prime}=-1.4 \cdot 10^{-3}$,

$K_{\xi}^{A}=-30\left[\mathrm{~m}^{2} / \mathrm{s}^{2}\right], K_{t \xi}^{A}=-10^{-3}\left[\mathrm{~m}^{2} /\left(\mathrm{s}^{2} \cdot \mathrm{K}\right)\right]$.

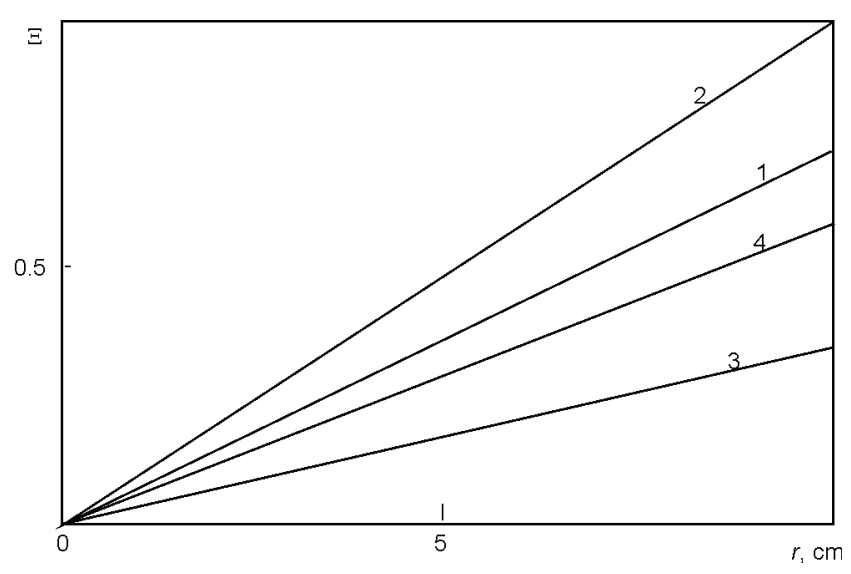

Fig. 1. Distribution of martensite after torsion 
Table 1. Distribution of martensite after torsion

\begin{tabular}{c|l|l|l|l}
\hline \multirow{2}{*}{$r[\mathrm{~cm}]$} & \multicolumn{4}{|c}{$\Xi^{*}$} \\
\cline { 2 - 5 } & NiTi & NiAl & CuAlNi & CuZn \\
\hline 0 & 0 & 0 & 0 & 0 \\
1 & 0.064 & 0.100 & 0.027 & 0.052 \\
2 & 0.128 & 0.199 & 0.054 & 0.104 \\
3 & 0.192 & 0.299 & 0.081 & 0.156 \\
4 & 0.256 & 0.398 & 0.108 & 0.208 \\
5 & 0.320 & 0.498 & 0.135 & 0.260 \\
6 & 0.384 & 0.597 & 0.162 & 0.312 \\
7 & 0.448 & 0.697 & 0.189 & 0.364 \\
8 & 0.512 & 0.796 & 0.216 & 0.416 \\
9 & 0.576 & 0.896 & 0.243 & 0.468 \\
10 & 0.640 & 0.995 & 0.270 & 0.520 \\
\hline
\end{tabular}

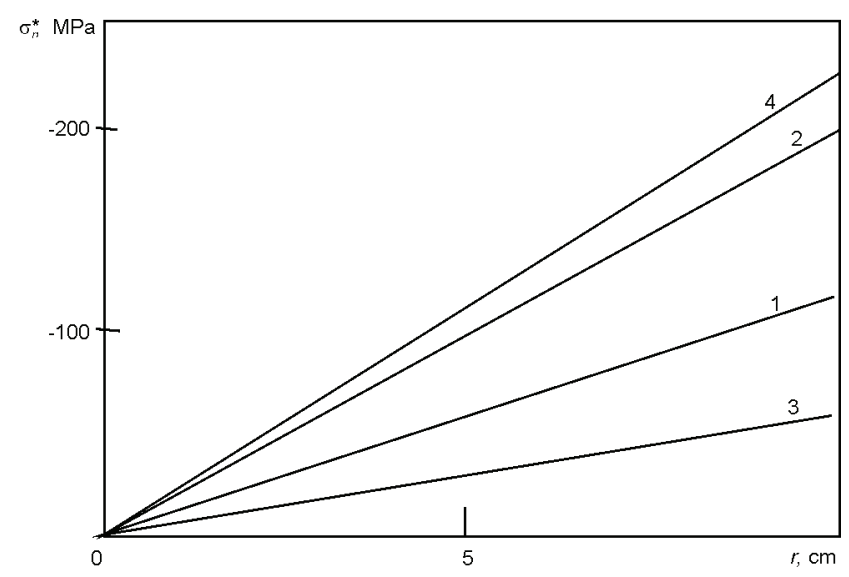

Fig. 2. Distribution of residual stresses due to torsion

Table 2. Distribution of residual stresses due to torsion

\begin{tabular}{c|c|c|c|c}
\hline \multirow{2}{*}{$\mathrm{r}[\mathrm{cm}]$} & \multicolumn{4}{|c}{$\sigma_{r}^{*}[\mathrm{MPa}]$} \\
\cline { 2 - 5 } & $\mathrm{NiTi}$ & $\mathrm{NiAl}$ & $\mathrm{CuAlNi}$ & $\mathrm{CuZn}$ \\
\hline 0 & 0 & 0 & 0 & 0 \\
1 & -11.100 & -20.249 & -4.230 & -21.176 \\
2 & -22.200 & -40.498 & -8.460 & -42.352 \\
3 & -33.300 & -60.747 & -12.690 & -63.528 \\
4 & -44.400 & -80.996 & -16.920 & -84.704 \\
5 & -55.500 & -101.245 & -21.150 & -105.880 \\
6 & -66.600 & -121.494 & -25.380 & -127.056 \\
7 & -77.700 & -141.743 & -29.610 & -148.232 \\
8 & -88.800 & -161.992 & -33.840 & -169.408 \\
9 & -99.900 & -182.241 & -38.070 & -190.584 \\
10 & -111.000 & -202.490 & -42.300 & -211.760 \\
\hline
\end{tabular}

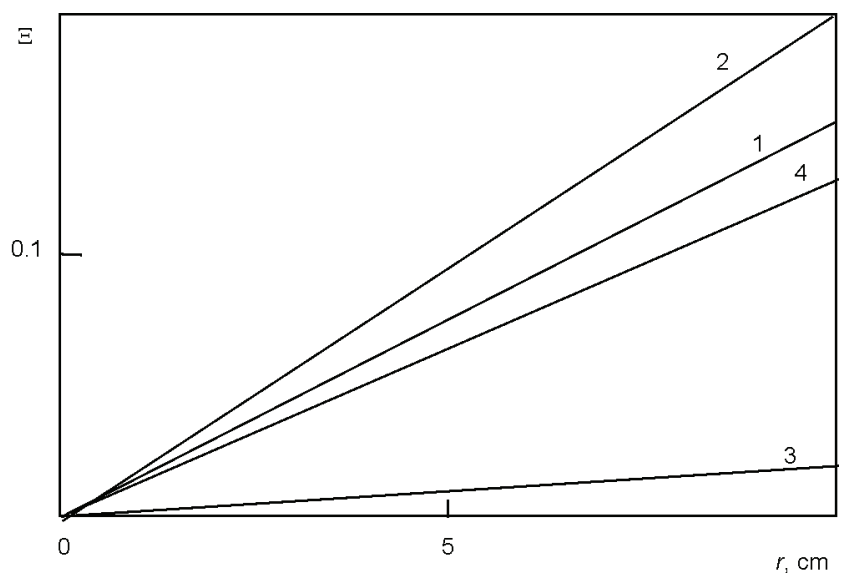

Fig. 3. Distribution of martensite after heating

Table 3. Distribution of martensite after heating

\begin{tabular}{c|c|c|cc}
\hline \multirow{2}{*}{$r[\mathrm{~cm}]$} & \multicolumn{4}{|c}{$\Xi$} \\
\cline { 2 - 5 } & NiTi & NiAl & CuAlNi & $\mathrm{CuZn}$ \\
\hline 0 & 0 & 0 & 0 & 0 \\
1 & 0.014 & 0.021 & 0.002 & 0.012 \\
2 & 0.028 & 0.041 & 0.004 & 0.024 \\
3 & 0.042 & 0.062 & 0.006 & 0.036 \\
4 & 0.056 & 0.082 & 0.008 & 0.048 \\
5 & 0.070 & 0.103 & 0.010 & 0.060 \\
6 & 0.084 & 0.123 & 0.012 & 0.072 \\
7 & 0.098 & 0.144 & 0.014 & 0.084 \\
8 & 0.112 & 0.164 & 0.016 & 0.096 \\
9 & 0.126 & 0.185 & 0.018 & 0.108 \\
10 & 0.140 & 0.205 & 0.020 & 0.120 \\
\hline
\end{tabular}

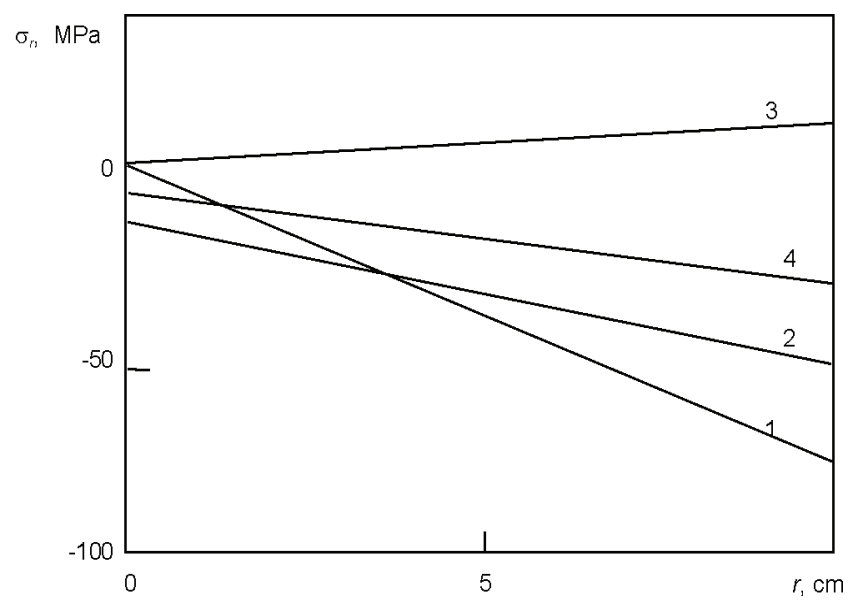

Fig. 4. Distribution of stresses after heating 
Table 4. Distribution of stresses after heating

\begin{tabular}{c|c|c|r|c}
\hline \multirow{2}{*}{$\mathrm{r}[\mathrm{cm}]$} & \multicolumn{4}{|c}{$\sigma_{r}[\mathrm{MPa}]$} \\
\cline { 2 - 5 } & \multicolumn{1}{|c|}{$\mathrm{NiTi}$} & \multicolumn{1}{c}{$\mathrm{NiAl}$} & $\mathrm{CuAlNi}$ & \multicolumn{1}{c}{$\mathrm{CuZn}$} \\
\hline 0 & -7.800 & -9.450 & -2.200 & -13.100 \\
1 & -15.000 & -12.399 & -1.730 & -16.406 \\
2 & -22.200 & -15.348 & -1.260 & -19.712 \\
3 & -29.400 & -18.297 & -0.790 & -23.018 \\
4 & -36.600 & -21.246 & -0.320 & -26.324 \\
5 & -43.800 & -24.195 & 0.150 & -29.630 \\
6 & -51.000 & -27.144 & 0.620 & -32.936 \\
7 & -58.200 & -30.093 & 1.090 & -36.242 \\
8 & -65.400 & -33.042 & 1.560 & -39.548 \\
9 & -72.600 & -35.991 & 2.030 & -42.854 \\
10 & -79.800 & -38.940 & 2.500 & -46.160 \\
\hline
\end{tabular}

\section{CONCLUSIONS}

Numerical investigations show that in the rod subjected to torsion. the non-uniform phase structure and the nonuniform distribution of residual stresses caused by it are formed. After subsequent heating the stresses are reduced which can be explained as a result of reverse transformation and the phase structure approaching its initial value. It corresponds with the known data about the behaviour of shape memory alloys $[6,7]$.
The developed model can serve as a theoretical basis for development of optimum modes of manufacturing and operation of constructive elements made from shape memory alloys.

\section{References}

[1] I. Goldenblat, Nonlinear problems of elasticity theory (in Russian), Nauka, Moscow (1969).

[2] V. Astashkin, O. Hachkevych, O. Onyshko, B. Bozhenko, Modeling of thermomechanical processes in deformable solids allowing for structure transformation with use of stress and strain tensor invariants (in Ukrainian). Mechanical Engineering 11, 14-17 (2003).

[3] V. Astashkin, B. Bozhenko, S. Budz, O. Onyshko, Modelling of thermomechanical processes in solids with use of stress and strain tensor invariants under technological heating (in Russian), Technological processes and systems designing. Societas Scientarium Lublinensis, Lublin, 164-170 (2003).

[4] O. Onyshko, B. Bozhenko, V. Novatskii, Model of thermomecanical phenomena in shape memory alloys (in Ukrainian), Mathematical problems of mechanics of nonhomogeneous structures, Lviv, 71-73 (2003).

[5] V.I. Astashkin, S.F. Budz, O.E. Onyshko, Quantitative description of physicomechanical processes in shape memory alloys, Materials Science 4, 453-459 (1994).

[6] V.A. Likhachov, S.L. Kuzmin, Z.P. Kamentseva, Shape memory effect (in Russian), Publishing house of Leningrad University, Leningrad (1987).

[7] H. Funakubo, Shape memory alloys (in Russian), Metallurgy, Moscow (1990).

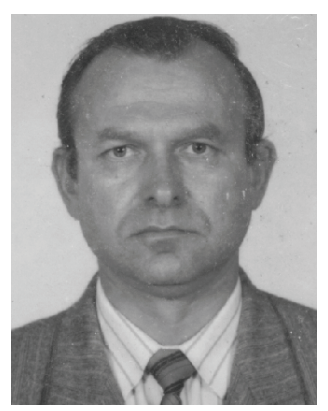

Dr. Volodymyr Astashin was born on November 25, 1951. He graduated in Physics of Metals at Lviv National Technical University in 1974. Since then he has been working at the Pidstryhach Institute for Applied Problems of Mechanics and Mathematics NASU in Lviv, Ukraine. He obtained the PhD degree in Mathematics in 1983. His research interests concern the deformable solid mechanics and mathematical modeling in thermomechanics of solids, especially subjected to structure changes. He is author or co-author of over 80 papers in major professional journals and conference proceedings.

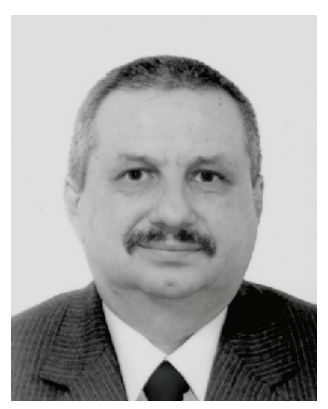

Dr. OLEKSII ONYShKo was born on January 31, 1955. He graduated in Mechanics at Lviv National University in 1976. Since then he has been working at the Pidstryhach Institute for Applied Problems of Mechanics and Mathematics NASU in Lviv, Ukraine. He obtained the PhD degree in Mathematics in 1985. His research interests concern the deformable solid mechanics and mathematical modeling in thermomechanics of solids, particularly subjected to structure changes. He is author or co-author of 60 papers in major professional journals and conference proceedings. 

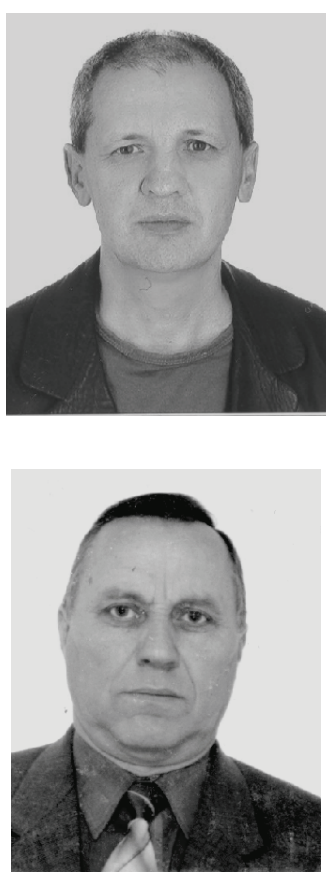

DR. BOHDAN BozHenko was born in Lviv, Ukraine, in 1956. Education: Lviv University - MSc in Applied Mathematic (1978), Pidstryhach Institute for Applied Problems of Mechanics and Mathematics National Ukrainian Academy of Sciences - PhD in Mechanics of Deformable Bodies (1984). Since 1996 has been working at the Institute of Physics and Mathematics at Opole Technical University. His research interests include mathematical modelling of physico-mechanical processes in inhomogeneous structures, computational methods. He is author or co-author of over 50 papers in major professional journals and conference proceedings.

DR. STEPAN BUDZ was born on October 17, 1940. He graduated in Mechanics at Lviv National University in 1965. Since then he has been working at the Pidstryhach Institute for Applied Problems of Mechanics and Mathematics NASU in Lviv, Ukraine. He obtained the PhD degree in Mathematics in 1975. He is the State Prize laureate of 1981. His research interests concern the deformable solid mechanics and optimization theory of thermomechanical processes in solids, particularly subjected to structure changes. He is author or co-author of over 90 papers in major professional journals and conference proceedings. 